\title{
O impacto da tontura na qualidade de vida de indivíduos com migrânea
}

\author{
The impact of dizziness on the quality of life of individuals with \\ migraine
}

\author{
Richelle Corrêa Sá Fortes ${ }^{1}$, Joice Souza Vicente ${ }^{2}$, Bianca Pinheiro Lanzetta ${ }^{3}$
}

\begin{abstract}
RESUMO
Objetivo: Caracterizar a interferência da tontura na qualidade de vida de adultos com migrânea qualificando seus efeitos impostos no cotidiano destes indivíduos nos aspectos físico, emocional e funcional, por meio do questionário DHI (Dizziness Handicap Inventory) Versão Brasileira. Métodos: Foram avaliados 25 pacientes adultos com diagnóstico de migrânea, de ambos os gêneros, com idades variando entre 20 e 80 anos. Os pacientes que apresentaram queixa de tontura responderam ao questionário DHI Versão Brasileira, que avalia a interferência da tontura na qualidade de vida dos pacientes, abordando os aspectos emocional, físico e funcional. Resultados: Dos 25 pacientes com migrânea entrevistados, $80 \%$ apresentaram queixa de tontura, dos quais $90 \%$ eram do gênero feminino. Os aspectos funcionais e físicos tiveram frequências de respostas semelhantes, comparadas entre si, diferenciando-se do emocional, que gerou mais inconsistências de respostas, além de ter apresentado um menor índice das mesmas, sendo este o aspecto que menos afeta a vida desses pacientes. Conclusão: $\mathrm{O}$ aspecto funcional foi o mais prejudicado na qualidade de vida dos pacientes pesquisados, seguido do aspectos físico e emocional, respectivamente. O DHI mostrou-se um instrumento fundamental como complemento na avaliação e no acompanhamento da evolução clínica desses indivíduos.
\end{abstract}

Descritores: Migrânea; Cefaleia; Tontura; Vertigem; Questionários; Qualidade de vida

\section{INTRODUÇÃO}

A cefaleia, popularmente conhecida como dor de cabeça, é uma desordem muito comum na humanidade, em que $90 \%$ dos indivíduos apresentam pelo menos um episódio por ano. Ela pode ser desencadeada por várias causas, sendo a migrânea a mais comum delas ${ }^{(1)}$.

Esta é uma desordem hereditária que desestabiliza a circulação cerebral no complexo arterial vertebrobasilar e apresenta elevada prevalência de alterações cocleovestibulares (tontura, zumbidos, plenitude auricular, fonofobia), com aparecimento de dores de cabeça recorrentes, em geral com caráter pulsátil, unilateral, precedida ou não de sintomas neurológicos focais, denominados de aura, e também hiperatividade, depressão, bocejos repetidos e dificuldade de memória ${ }^{(2)}$.

Trabalho realizado na Escola Superior de Ciências da Santa Casa de Misericórdia de Vitória - EMESCAM - Vitória (ES), Brasil.

(1) Especialista em Audiologia, Fonoaudióloga da Prefeitura Municipal de Vitória - Vitória (ES), Brasil.

(2) Especialista em Audiologia, Fonoaudióloga da Clínica Multifisio - Vitória (ES), Brasil.

(3) Mestre, Fonoaudióloga da Policlínica de Referência do Centro Universitário Vila Velha - UVV - Vila Velha (ES), Brasil.

Endereço para correspondência: Richelle Corrêa Sá Fortes. R. João Nunes Coelho, 236/301, Mata da Praia, Vitória (ES), Brasil, CEP: 29065-490. E-mail: richellecsf@hotmail.com

Recebido em: 15/9/2009; Aceito em: 23/03/2010
Ela é caracterizada por um conjunto de sintomas neurológicos como cefaleia, náuseas, vômitos, tontura, entre outros que, por sua vez, compromete a qualidade de vida de muitos indivíduos no âmbito social, emocional, no desempenho do trabalho e também nos estudos e formação acadêmica. Ela pode aparecer em indivíduos de todas as idades e seu grau de manifestação pode ser determinado por fatores genéticos e de gênero ${ }^{(3)}$.

As alterações cocleovestibulares, de origem periférica ou central, podem ser justificadas por isquemias que levam a infartos no tecido nervoso na fase de vasoconstrição da crise migranosa e podem também ter relação com a vasoconstrição dos ramos das artérias vertebral e basilar que irrigam o nervo cocleovestibular e seus ramos, e o vestíbulo ${ }^{(4)}$.

O quadro vertiginoso de curta duração, com cefaleia, pode ter relação com vasoespasmos transitórios do sistema vertebrobasilar ou ainda com a despolarização de células neurais e gliais com intensa atividade neural que dura cerca de 60 segundos, seguida de um silêncio neural por alguns minutos ${ }^{(5)}$.

A vertigem de longa duração e/ou náuseas relacionadas a movimentos cefálicos, com ou sem cefaleia, relacionam-se à liberação de neuropeptídeos nas estruturas vestibulares periféricas e centrais, com aumento da excitabilidade de receptores vestibulares na orelha interna e elevação da sensibilidade a movimentos cefálicos ${ }^{(5)}$. 
A vertigem pode ocorrer antes, durante, após a fase álgica da migrânea ou nos intervalos intercrise. A vertigem que surge um pouco antes da cefaleia pode ser considerada um tipo de aura da migrânea ${ }^{(5)}$.

A migrânea pode ser classificada por: migrânea clássica, com aura; comum, sem aura; e os chamados "equivalentes migranosos", que são a vertigem paroxística benigna da infância, a enxaqueca basilar e a vertigem recidivante benigna no adulto ${ }^{(6)}$.

A clássica, com aura, apresenta sintomas neurológicos temporários que consistem em disfunções sensoriais, motoras ou cognitivas, que geralmente precedem a cefaleia ou aparecem durante ou após o acesso. A comum, sem aura, caracteriza-se por cefaleias periódicas que geralmente são pulsáteis e unilaterais, exacerbadas por atividades e associadas com a náusea, a fotofobia e a fonofobia ${ }^{(7)}$. A migrânea com ou sem aura, frequentemente, começa entre os 12 e os 30 anos de idade, e após os 50 é menos comum e costuma apresentar-se como uma aura de migrânea sem cefaleia ${ }^{(7)}$.

A vertigem e o desequilíbrio ligados à migrânea geralmente respondem ao mesmo tipo de tratamento proposto para as cefaleias migranosas. O seu tratamento pode ser dividido em redução dos fatores de risco, como o estresse, a ansiedade, a hipoglicemia, a flutuação estrogênica, certos alimentos e tabagismo; tratamento médico abortivo, onde são utilizadas drogas projetadas para abortar a cefaleia; e tratamento médico profilático, diário, designado para preveni-la ${ }^{(7)}$.

Os sintomas cocleovestibulares causados pela migrânea podem ter grande interferência na qualidade de vida dos indivíduos. Dentre eles a tontura, caracterizada por sensação de alteração do equilíbrio corporal, podendo ser de caráter rotatório (vertigem) ou não-rotatório (oscilação, instabilidade, flutuação, etc) ${ }^{(8)}$.

Esta, quando frequente, interfere de maneira negativa nas atividades diárias do indivíduo, principalmente nos níveis emocional, físico e funcional, diminuindo assim sua qualidade de $\operatorname{vida}^{(9)}$.

A Organização Mundial de Saúde (OMS) define a qualidade de vida como "a percepção do sujeito de sua posição na vida, no contexto de sua cultura e sistema de valores nos quais ele está inserido e em relação aos seus objetivos, expectativas, padrões e preocupações"(10).

Um meio capaz de definir um perfil detalhado do indivíduo vertiginoso sobre o quanto sua qualidade de vida encontrase alterada é o Dizziness Handicap Inventory (DHI) ${ }^{(2)}$. Este questionário específico para tontura, elaborado por Jacobson e Newman ${ }^{(11)}$, é composto de 25 questões que avaliam a autopercepção dos efeitos incapacitantes impostos pela tontura (aspectos emocionais, físicos e funcionais). O DHI é o único questionário que foi traduzido e adaptado para aplicação na população brasileira ${ }^{(12)}$.

O objetivo deste trabalho foi caracterizar o quanto a tontura interfere na qualidade de vida de adultos com migrânea, qualificando seus efeitos impostos nos aspectos físico, emocional e funcional, no cotidiano destes indivíduos, por meio do questionário DHI Versão Brasileira.

\section{MÉTODOS}

Este estudo foi realizado na cidade de Vitória (ES), no
Ambulatório de Cefaleia da Escola Superior de Ciências da Santa Casa de Misericórdia de Vitória (EMESCAM), com 25 adultos com diagnóstico de migrânea e em fase variada de tratamento, e constou da aplicação de um questionário, o DHI Versão Brasileira, nos 20 adultos do grupo que apresentaram queixa de tontura.

Foram incluídos neste estudo os pacientes que apresentaram: idade entre 20 e 80 anos, diagnóstico de migrânea, e queixa de tontura.

Foram excluídos deste estudo os pacientes que apresentaram alterações psicológicas que impedissem de compreender a execução da avaliação.

Todos os pacientes assinaram termo de consentimento livre e esclarecido e o projeto inicial foi submetido ao Comitê de Ética do Centro Universitário Vila Velha - $\mathrm{n}^{\circ}$ protocolo 84/2008, que o aprovou sem ressalvas.

A primeira etapa do estudo deu-se na avaliação neurológica, com a seleção dos pacientes com migrânea após o fechamento de diagnóstico. O diagnóstico de migrânea foi realizado de acordo com os critérios da Classificação Internacional das Cefaléias ${ }^{(13)}$. Todos os pacientes avaliados preencheram os critérios para diagnóstico de Migrânea ou Provável Migrânea. Em seguida, realizou-se a aplicação do DHI Versão Brasileira, individualmente, no próprio Ambulatório de Cefaleia.

O questionário DHI Versão Brasileira consiste em 25 questões que avaliam a interferência da tontura na qualidade de vida dos pacientes, abordando os aspectos emocional, físico e funcional, como mostra o Quadro 1.

Para análise dos resultados, o DHI considerada três possibilidades de respostas: sim, correspondendo a 4 pontos; às vezes, 2 pontos; e não, 0 pontos. A pontuação máxima do aspecto emocional é de 32 pontos, do físico 36 pontos e funcional 32 pontos, totalizando um escore de 100 pontos. Quanto maior o escore do DHI, mais prejudicada a qualidade de vida do paciente.

A análise realizada neste trabalho consistiu na exploração dos dados utilizando as técnicas de estatística descritiva (distribuição de frequências) e estatística inferencial (intervalo de confiança e teste de hipótese) para as variáveis estudadas. Foi utilizado o programa computacional SPSS 11.5 for Windows.

\section{RESULTADOS}

\section{Estatística descritiva}

Dos 25 pacientes migranosos selecionados para estudo, 20 (80\%) apresentaram tontura. Dentre os indivíduos com tontura, $18(90 \%)$ eram do gênero feminino e dois (10\%) do gênero masculino. Quanto à faixa etária, cinco $(25 \%)$ apresentavam entre 20 e 40 anos de idade; $13(65 \%)$ entre 41 e 60 anos de idade; e dois (10\%) entre 61 e 80 anos de idade.

Em relação ao tempo de tratamento da migrânea, quatro (20\%) dos pacientes encontraram-se em um período de até seis meses; quatro (20\%) entre seis meses a dois anos de tratamento; cinco (25\%) no período entre dois a quatro anos; cinco $(25 \%)$ entre quatro até seis anos de tratamento; e dois $(10 \%)$ no período maior que seis anos de tratamento.

Dentre os aspectos questionados, os funcionais e físicos 
Quadro 1. Dizziness Handicap Inventory - DHI Versão Brasileira

\begin{tabular}{|c|c|c|c|}
\hline Questões & Sim & Às vezes & Não \\
\hline \multicolumn{4}{|l|}{ O-1 Olhar para cima aumenta seu problema? } \\
\hline \multicolumn{4}{|l|}{ E-2 Sente-se frustrado (a) devido ao seu problema? } \\
\hline \multicolumn{4}{|l|}{ F-3 Por causa do seu problema, você restringe as viagens de trabalho ou recreação? } \\
\hline \multicolumn{4}{|l|}{ O-4 Andar no corredor de um supermercado aumenta seu problema? } \\
\hline \multicolumn{4}{|l|}{ F-5 Devido ao seu problema, você tem dificuldade para se deitar ou levantar da cama? } \\
\hline \multicolumn{4}{|l|}{$\begin{array}{l}\text { F-6 O seu problema restringe muito a sua participação em atividades sociais como sair para jantar, ir ao } \\
\text { cinema, dançar ou ir às festas? }\end{array}$} \\
\hline \multicolumn{4}{|l|}{ F-7 Por causa do seu problema, você tem dificuldade para ler? } \\
\hline \multicolumn{4}{|l|}{$\begin{array}{l}\text { O-8 A execução de atividades como esportes, dança, pequenas tarefas caseiras como varrer ou retirar } \\
\text { os pratos aumenta seu problema? }\end{array}$} \\
\hline \multicolumn{4}{|l|}{ E-9 Você tem medo de sair de casa sem que alguém o (a) acompanhe por causa do seu problema? } \\
\hline \multicolumn{4}{|l|}{ E-10 Você se sente envergonhado (a) frente a outras pessoas por causa do seu problema? } \\
\hline \multicolumn{4}{|l|}{ O-11 Movimentos rápidos de cabeça aumentam seu problema? } \\
\hline \multicolumn{4}{|l|}{ F-12 Você evita alturas devido ao seu problema? } \\
\hline \multicolumn{4}{|l|}{ O-13 Virar-se na cama aumenta seu problema? } \\
\hline \multicolumn{4}{|l|}{ F-14 É difícil para você realizar trabalhos caseiros? } \\
\hline \multicolumn{4}{|l|}{ E-15 Por causa do seu problema você tem medo que as pessoas pensem que está drogado (a)? } \\
\hline \multicolumn{4}{|l|}{ O-16 É difícil caminhar sozinho (a) por causa do seu problema? } \\
\hline \multicolumn{4}{|l|}{ O-17 Andar numa calçada aumenta seu problema? } \\
\hline \multicolumn{4}{|l|}{ F-18 Você sente dificuldade para se concentrar por causa do seu problema? } \\
\hline \multicolumn{4}{|l|}{ O-19 Por causa do seu problema é difícil para você andar ao redor da casa no escuro? } \\
\hline \multicolumn{4}{|l|}{ E-20 Por causa do seu problema você tem medo de ficar sozinho (a) em casa? } \\
\hline \multicolumn{4}{|l|}{ E-21 Você se sente prejudicado por causa do seu problema? } \\
\hline \multicolumn{4}{|l|}{ E-22 O seu problema provocou tensão em seu relacionamento com familiares ou amigos? } \\
\hline \multicolumn{4}{|l|}{ E-23 Você se sente deprimido (a) por causa do seu problema? } \\
\hline \multicolumn{4}{|l|}{ F-24 O seu problema interfere no trabalho ou em suas responsabilidades nos afazeres domésticos? } \\
\hline O - 25 Inclinar-se piora seu problema? & & & \\
\hline
\end{tabular}

Legenda: $\mathrm{O}$ = aspecto físico; $\mathrm{E}=$ aspecto emocional; $\mathrm{F}$ = aspecto funcional

Fonte: Castro ASO, Gazzola JM, Natour J, Ganança FF. Versão brasileira do Dizziness Handicap Inventory. Pró-Fono. 2007;19(1):97-104.(12)

foram os mais prejudicados, seguido do emocional. A Tabela 1 mostra os escores totais dos três aspectos.

As Tabelas 2, 3 e 4 mostram a distribuição das frequências de respostas de todos os pacientes segundo os aspectos físico, emocional e funcional, respectivamente.

$\mathrm{Na}$ estatística descritiva dos aspectos avaliados no DHI Versão Brasileira e o escore total, verifica-se que a pontuação máxima foi igual para os aspectos físico e funcional; a menor pontuação média foi a do aspecto emocional. A variabilidade das pontuações dos aspectos físico e funcional foi semelhante ( $C V=42,1 \%$ e $C V=40,8 \%$ respectivamente), e pode-se verificar também que foram semelhantes ao escore geral $(\mathrm{CV}=40,2 \%)$, enquanto que houve alta variabilidade das pontuações do aspecto Emocional $(\mathrm{CV}=64,3 \%)$.

\section{Estatística inferencial}

Na Tabela 5, para a comparação entre essas duas categorias, foi utilizado o teste de médias t-Student para amostras independentes, com nível de significância de 5\%. Observa-se que houve diferença estatística entre os aspectos físico e emocional e entre os aspectos emocional e funcional versus emocional e versus funcional, a hipótese entre as médias $\left(\mathrm{H}_{0}\right)$ foi rejeitada, ou seja, houve diferença entre os aspectos testados, portanto, as médias são iguais, enquanto que para entre o aspecto físico versus funcional, não houve diferença estatisticamente entre os aspectos testados, portanto, as médias são diferentes.

De acordo com a Figura 1, o número médio de pontuações foi semelhante para os aspectos físico e funcional, enquanto que para o aspecto emocional a pontuação média foi menor.

\section{DISCUSSÃO}

Dos 25 indivíduos selecionados para estudo, 20 apresentaram queixa de tontura e nortearam o objetivo deste estudo, no qual foi caracterizou-se o quanto a tontura interfere na qualidade de vida de adultos com migrânea. A seleção desses indivíduos ocorreu de acordo com os critérios de inclusão e exclusão dessa pesquisa.

Dentre os indivíduos estudados, a prevalência da migrânea no gênero feminino foi muito significativa, $90 \%$, condizendo com alguns estudos que também apresentam este dado ${ }^{(1,14,15)}$. Isso pode ser explicado pelos fatores clínicos relacionados às variações dos hormônios femininos ${ }^{(1,15,16)}$. 
Tabela 1. Escores dos aspectos Físicos, Emocionais e Funcional obtido à aplicação do DHI Versão Brasileira

\begin{tabular}{|c|c|c|c|c|}
\hline $\begin{array}{l}\text { Identificação } \\
\text { do paciente }\end{array}$ & 0 & $E$ & $F$ & Total \\
\hline 1 & 28 & 12 & 22 & 62 \\
\hline 2 & 28 & 14 & 24 & 66 \\
\hline 3 & 22 & 16 & 24 & 62 \\
\hline 4 & 22 & 18 & 32 & 72 \\
\hline 5 & 20 & 4 & 8 & 32 \\
\hline 6 & 22 & 28 & 30 & 80 \\
\hline 7 & 20 & 0 & 14 & 34 \\
\hline 8 & 24 & 18 & 20 & 62 \\
\hline 9 & 32 & 24 & 28 & 84 \\
\hline 10 & 14 & 8 & 22 & 44 \\
\hline 11 & 12 & 14 & 40 & 66 \\
\hline 12 & 16 & 18 & 14 & 48 \\
\hline 13 & 0 & 4 & 4 & 8 \\
\hline 14 & 12 & 10 & 28 & 50 \\
\hline 15 & 6 & 12 & 28 & 46 \\
\hline 16 & 26 & 16 & 28 & 70 \\
\hline 17 & 30 & 10 & 20 & 60 \\
\hline 18 & 12 & 0 & 6 & 18 \\
\hline 19 & 24 & 30 & 32 & 86 \\
\hline 20 & 24 & 26 & 30 & 80 \\
\hline Total & 394 & 278 & 428 & 1100 \\
\hline
\end{tabular}

Legenda: $\mathrm{O}=$ aspecto físico; $\mathrm{E}=$ aspecto emocional; $\mathrm{F}=$ aspecto funcional

A tontura é um sintoma frequente em pacientes migranosos, como foi demonstrado neste estudo, em que $80 \%$ dos pacientes entrevistados apresentaram esta queixa. Estudo anterior, com 500 pacientes adultos migranosos já havia constatado que a tontura acomete $72 \%$ deles $^{(1)}$.

Por ser um sintoma que interfere muito na qualidade de vida das pessoas, o DHI Versão Brasileira torna-se um instrumento de avaliação importante para profissionais da saúde envolvidos no tratamento destes pacientes, já que este qualifica os aspectos físico, funcional e emocional ${ }^{(2)}$.

Dentre os aspectos avaliados pelo DHI Versão Brasileira, o mais afetado foi o aspecto funcional, em que muitos pacientes relataram limitações quanto às atividades sociais. As funções mais prejudicadas foram evitar altura, dificuldade em ler e dificuldade para se deitar ou levantar da cama.

Essas alterações vestibulares geram restrições em atividades tanto físicas como sociais, o que afeta negativamente as relações sociais ${ }^{(17)}$. Não foi encontrado na literatura nenhum estudo específico relacionando a tontura e o impacto desta na qualidade de vida dos indivíduos migranosos que pudesse servir como parâmetro de comparação para esta pesquisa.

Neste estudo, o segundo aspecto mais afetado foi o físico. Indivíduos que apresentam migrânea associada à disfunção vestibular geralmente apresentam vertigem espontânea ou de posição, e ainda, ela pode começar como espontânea e transformar-se em posicional ${ }^{(18)}$. Uma característica marcante desse tipo de migrânea é a intolerância a movimentos do próprio corpo ou do ambiente ${ }^{(19)}$, isso justifica as queixas dos pacientes nas quais as funções mais prejudicadas foram movimentos rápidos de cabeça, inclinar-se e olhar para cima.

Nos aspectos emocionais, os pacientes relataram sentir-se muito prejudicados com o seu problema causando, em alguns casos, depressão e frustração.

Autores apontam a depressão, a ansiedade e o medo como os sintomas psicológicos co-mórbidos da vertigem, tornando o prognóstico menos positivo, com consequente defasagem na qualidade de vida desses pacientes ${ }^{(20)}$. Indivíduos migranosos apresentam risco de sofrer depressão três vezes maior do que pacientes sem cefaleia, assim como duas vezes mais risco de ansiedade, sugerindo a ocorrência de mecanismos patológicos comuns $^{(21,22)}$.

As respostas referentes aos aspectos físicos e funcionais foram muito semelhantes em relação à média e variabilidade,

Tabela 2. Distribuição de frequências segundo o aspecto Físico

\begin{tabular}{|c|c|c|c|c|c|c|c|c|}
\hline \multirow{2}{*}{ Aspecto físico } & \multicolumn{2}{|l|}{ Sim } & \multicolumn{2}{|l|}{ Não } & \multicolumn{2}{|c|}{ Às vezes } & \multicolumn{2}{|l|}{ Total } \\
\hline & Frequência & $\%$ & Frequência & $\%$ & Frequência & $\%$ & Frequência & $\%$ \\
\hline Olhar para cima aumenta seu problema & 13 & 65 & 1 & 5 & 6 & 30 & 20 & 100 \\
\hline $\begin{array}{l}\text { Andar no corredor de um supermercado } \\
\text { aumenta seu problema }\end{array}$ & 9 & 45 & 3 & 15 & 8 & 40 & 20 & 100 \\
\hline $\begin{array}{l}\text { A execução de atividades como esportes, dança, } \\
\text { pequenas tarefas caseiras como varrer ou retirar } \\
\text { os pratos aumenta seu problema }\end{array}$ & 6 & 30 & 5 & 25 & 9 & 45 & 20 & 100 \\
\hline $\begin{array}{l}\text { Movimentos rápidos de cabeça aumentam seu } \\
\text { problema }\end{array}$ & 18 & 90 & 1 & 5 & 1 & 5 & 20 & 100 \\
\hline Virar-se na cama aumenta seu problema & 4 & 20 & 2 & 10 & 14 & 70 & 20 & 100 \\
\hline $\begin{array}{l}\text { É difícil caminhar sozinho (a) por causa do } \\
\text { problema }\end{array}$ & 7 & 35 & 1 & 5 & 12 & 60 & 20 & 100 \\
\hline Andar numa calçada aumenta seu problema & 10 & 50 & 3 & 15 & 7 & 35 & 20 & 100 \\
\hline $\begin{array}{l}\text { Por causa do seu problema é difícil para você } \\
\text { andar ao redor da casa no escuro }\end{array}$ & 9 & 45 & 2 & 10 & 9 & 45 & 20 & 100 \\
\hline Inclinar-se piora seu problema & 14 & 70 & 1 & 5 & 5 & 25 & 20 & 100 \\
\hline
\end{tabular}


Tabela 3. Distribuição de frequências segundo o aspecto Emocional

\begin{tabular}{|c|c|c|c|c|c|c|c|c|}
\hline \multirow{2}{*}{ Aspecto emocional } & \multicolumn{2}{|l|}{ Sim } & \multicolumn{2}{|l|}{ Não } & \multicolumn{2}{|c|}{ Às vezes } & \multicolumn{2}{|l|}{ Total } \\
\hline & Frequência & $\%$ & Frequência & $\%$ & Frequência & $\%$ & Frequência & $\%$ \\
\hline Sente-se frustrado (a) devido ao seu problema & 11 & 55 & 2 & 10 & 7 & 35 & 20 & 100 \\
\hline $\begin{array}{l}\text { Você tem medo de sair de casa sem que alguém } \\
\text { o (a) acompanhe por causa do seu problema }\end{array}$ & 8 & 40 & 1 & 5 & 11 & 55 & 20 & 100 \\
\hline $\begin{array}{l}\text { Você se sente envergonhado (a) frente a outras } \\
\text { pessoas por causa do seu problema }\end{array}$ & 4 & 20 & 1 & 5 & 15 & 75 & 20 & 100 \\
\hline $\begin{array}{l}\text { Por causa do seu problema você tem medo que } \\
\text { as pessoas pensem que está drogado (a) }\end{array}$ & - & - & 2 & 10 & 18 & 90 & 20 & 100 \\
\hline $\begin{array}{l}\text { Por causa do seu problema você tem medo de } \\
\text { ficar sozinho (a) em casa }\end{array}$ & 8 & 40 & 2 & 10 & 10 & 50 & 20 & 100 \\
\hline Você se sente prejudicado com o seu problema & 13 & 65 & 2 & 10 & 5 & 25 & 20 & 100 \\
\hline $\begin{array}{l}\text { O seu problema provocou tensão em seu } \\
\text { relacionamento com familiares e amigos }\end{array}$ & 7 & 35 & 2 & 10 & 11 & 55 & 20 & 100 \\
\hline $\begin{array}{l}\text { Você se sente deprimido (a) por causa do seu } \\
\text { problema }\end{array}$ & 12 & 60 & 2 & 10 & 6 & 30 & 20 & 100 \\
\hline
\end{tabular}

Tabela 4. Distribuição de frequências segundo o aspecto Funcional

\begin{tabular}{|c|c|c|c|c|c|c|c|c|}
\hline \multirow{2}{*}{ Aspecto funcional } & \multicolumn{2}{|l|}{ Sim } & \multicolumn{2}{|l|}{ Não } & \multicolumn{2}{|c|}{ Às vezes } & \multicolumn{2}{|l|}{ Total } \\
\hline & Frequência & $\%$ & Frequência & $\%$ & Frequência & $\%$ & Frequência & $\%$ \\
\hline $\begin{array}{l}\text { Por causa do seu problema restringe as viagens } \\
\text { de trabalho ou recreação }\end{array}$ & 12 & 60 & 1 & 5 & 7 & 30 & 20 & 100 \\
\hline $\begin{array}{l}\text { Devido ao seu problema, você tem dificuldade } \\
\text { para se deitar ou levantar da cama }\end{array}$ & 14 & 70 & 1 & 5 & 5 & 25 & 20 & 100 \\
\hline $\begin{array}{l}\text { O seu problema restringe muito a sua } \\
\text { participação em atividades sociais como sair } \\
\text { para jantar, ir ao cinema, dançar ou ir às festas }\end{array}$ & 11 & 55 & 1 & 5 & 8 & 40 & 20 & 100 \\
\hline $\begin{array}{l}\text { Por causa do seu problema, você tem dificuldade } \\
\text { em ler }\end{array}$ & 15 & 75 & 2 & 10 & 3 & 15 & 20 & 100 \\
\hline Você evita altura devido ao seu problema & 17 & 85 & 1 & 5 & 2 & 10 & 20 & 100 \\
\hline É difícil para você realizar trabalhos caseiros & 5 & 25 & 8 & 40 & 7 & 35 & 20 & 100 \\
\hline $\begin{array}{l}\text { Você sente dificuldade para se concentrar por } \\
\text { causa do seu problema }\end{array}$ & 13 & 65 & 2 & 10 & 5 & 25 & 20 & 100 \\
\hline $\begin{array}{l}\text { O seu problema interfere no trabalho ou em suas } \\
\text { responsabilidades nos afazeres domésticos }\end{array}$ & 11 & 55 & 3 & 15 & 6 & 30 & 20 & 100 \\
\hline
\end{tabular}

Tabela 5. Teste t para igualdade de médias para amostras independentes

\begin{tabular}{lccc}
\hline $\begin{array}{l}\text { Pontuação dos } \\
\text { aspectos }\end{array}$ & $\begin{array}{c}\text { Estatística de } \\
\text { teste } t\end{array}$ & Valor de $\mathrm{p}$ & Resultado \\
\hline $\begin{array}{l}\text { Físico } \mathrm{x} \\
\text { funcional }\end{array}$ & $-0,631$ & 0,531 & Não rejeita-se $\mathrm{H}_{0}$ \\
\hline $\begin{array}{l}\text { Físico } \mathrm{x} \\
\text { emocional }\end{array}$ & 2,128 & 0,040 & Rejeita-se $\mathrm{H}_{0}$ \\
\hline $\begin{array}{l}\text { Emocional } \mathrm{x} \\
\text { funcional }\end{array}$ & $-2,685$ & 0,011 & Rejeita-se $\mathrm{H}_{0}$ \\
\hline
\end{tabular}

ou seja, tiveram respostas parecidas em cada paciente, diferenciando-se do emocional, que obteve uma maior variabilidade.

De acordo com a análise do questionário aplicado, os aspectos funcionais e físicos foram os mais prejudicados, seguido do aspecto emocional.

O questionário DHI Versão Brasileira torna-se de suma importância como complemento na avaliação e também como método de acompanhamento da evolução clínica. Para um

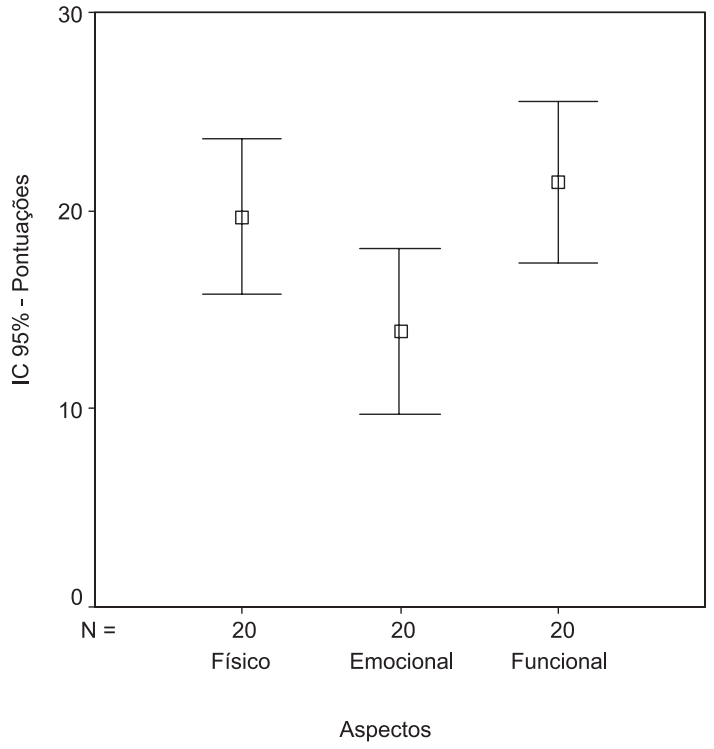

Figura 1. Médias para pontuações dos aspectos Físico, Emocional e Funcional 
bom prognóstico é necessário uma visão holística do paciente. Preocupar-se não só com a sua doença, mas com o que esta acarreta em sua qualidade de vida, contribui para resultados mais efetivos e satisfatórios mostrando a necessidade da interdisciplinaridade no tratamento desses pacientes, como por exemplo, neurologistas, otorrinolaringologistas, fonoaudiólogos, psicólogos, entre outros profissionais.

\section{CONCLUSÃO}

Os indivíduos com migrânea avaliados no presente estudo apresentaram alta prevalência de tontura. A aplicação do DHI
Versão Brasileira mostrou a interferência dessa alteração na sua qualidade de vida. $\mathrm{O}$ aspecto funcional foi o mais afetado, seguido de forma decrescente pelos aspectos físico, que teve resultado semelhante ao funcional, e o emocional, provocando assim, um grande impacto na vida social desses indivíduos, limitando suas atividades cotidianas.

\section{AGRADECIMENTOS}

Agradecemos pelo incentivo e apoio do Dr. Renan Barros Domingues e Dr. Carlos Kazuo Taguchi nesta pesquisa.

\begin{abstract}
Purpose: To characterize the interference of dizziness in the quality of life of adults with migraine by describing its effects on the physical, emotional and functional aspects of the daily life of these individuals, using the questionnaire DHI (Dizziness Handicap Inventory) Brazilian Version. Methods: Twenty-five adult patients of both genders, with ages between 20 and 80 years and diagnosis of migraine were evaluated. The subjects with dizziness complaints completed the DHI questionnaire Brazilian Version, which assesses the interference of dizziness in the quality of life of individuals, regarding physical, emotional and functional aspects. Results: From the 25 patients with migraine interviewed, $80 \%$ presented dizziness complaint, and $90 \%$ of these were women. Functional and physical aspects had similar responses, compared to each other, which were different from the emotional aspect, which presented more inconsistent answers and a lower score. The emotional aspect was the one that less affected the patients' lives. Conclusion: The functional aspect was the most impaired in the quality of life of the studied patients, followed by physical and emotional aspects, respectively. The DHI proved to be an essential instrument to complement patients' assessment and for the monitoring of their clinical evolution.
\end{abstract}

Keywords: Migraine; Headache; Dizziness; Vertigo; Questionnaires; Quality of life

\title{
REFERÊNCIAS
}

1. Raskin NH. Cefaleia. In: Kasper DL, Braunwald E, Fauci AS, Hauser SL, Longo DL, Jameson JL. Harrison medicina interna. 16a ed. Rio de Janeiro: McGraw-Hill; 2006. Vol. 1. p. 91-5.

2. Ganança CF, Kuhn AMB, Caovilla HH, Ganança MM. Vertigem e migrânea: achados otoneurológicos na nistagmografia computadorizada e à auto-rotação cefálica. Acta ORL. 2005;23(4):165-8.

3. Ganança FF, Castro ASO, Branco FC, Natour J. Interferência da tontura na qualidade de vida de pacientes com síndrome vestibular periférica. Rev Bras Otorrinolaringol. 2004;70(1):94-101.

4. Ramos DFR, Bernardi APA, Mor R. Principais sintomas e resultados da vectoeletronistagmografia digital em sujeitos com queixa de migrânea. Rev CEFAC. 2006;8(3):393-401.

5. Albertino S. Migrâneas relacionadas às alterações cocleovestibulares. In: Ganança MM, Munhoz MSL, Caovilla HH, Silva MLG. Estratégias terapêuticas em otoneurologia. São Paulo: Atheneu; 2001. vol. 4. p. 131.

6. Bergano PS, Cabete CF, Gushiken P, Uehara I, Frazza MM, Caovilla $\mathrm{HH}$, et al. Achados otoneurológicos em indivíduos com migrânea. Acta AWHO. 2000;19(2):88-95.

7. Tusa RJ. Diagnóstico e tratamento das disfunções otoneurológicas devido à migrânea. In: Herdman SJ. Reabilitação vestibular. 2a ed. São Paulo: Manole; 2002. p. 294-304.

8. Ganança MM, Caovilla HH. A vertigem e sintomas associados. In: Ganança MM, Vieira RM, Caovilla HH, editores. Princípios de otoneurologia. São Paulo: Atheneu; 1998. p.3.

9. Santos JB, Garcia AP. Estudo do impacto da qualidade de vida de indivíduos portadores de tontura. Acta ORL. 2007;25(2):152-6.

10. The World Health Organization Quality of Life assessment (WHOQOL): position paper from the World Health Organization. Soc Sci Med. 1995;41(10):1403-9.

11. Jacobson GP, Newman CW. The development of the Dizziness Handicap Inventory. Arch Otolaryngol Head Neck Surg. 1990;116(4):424-7.
12. Castro ASO, Gazzola JM, Natour J, Ganança FF. Versão brasileira do Dizziness Handicap Inventory. Pró-Fono. 2007;19(1):97-104.

13. Headache Classification Subcommittee of the International Headache Society. The International Classification of Headache Disorders: 2nd edition. Cephalalgia. 2004;24 Suppl 1:9-160.

14. Domingues RB, Kuster GW, Dutra LA, Santos JG. Headache epidemiology in Vitória, Espírito Santo. Arq Neuropsiquiatr. 2004;62(3A):588-91

15. Pahim LS, Menezes AMB, Lima R. Prevalência e fatores associados à enxaqueca na população adulta de Pelotas, RS. Rev Saúde Pública = J Public Health. 2006;40(4):692-8.

16. Rasmussen BK. Migraine and tension-type headache in a general population: precipitating factors, female hormones, sleep pattern and relation to lifestyle. Pain. 1993;53(1):65-72.

17. Yardley L, Putman J. Quantitative analysis of factors contributing to handicap and distress in vertiginous patients: a questionnaire study. Clin Otolaryngology Allied Sci. 1992;17(3):231-6.

18. Cal R, Bahmad Júnior F. Enxaqueca associada à disfunção auditivovestibular. Rev Bras Otorrinolaringol. 2008;74(4):606-12.

19. Bordini CA. Neurootologia da migrânea. Migrâneas cefaleias. 2003;6(1):19-21.

20. Paiva AD, Kuhn AMB. Sintomas psicológicos concomitantes à queixa de vertigem em 846 prontuários de pacientes otoneurológicos do Ambulatório de Otoneurologia da Universidade Federal de São Paulo - Escola Paulista de Medicina. Rev Bras Otorrinolaringol. 2004;70(4):512-5.

21. Breslau N, Schultz LR, Stewart WF, Lipton RB, Lucia VC, Welch KM. Headache and major depression: is the association specific to migraine? Neurology. 2000;54(2):308-13.

22. Costa EC, Ybarra MI, Correa H, Teixeira AL. Enxaqueca e depressão: comorbidade ou espectro? RBM Rev Bras Med. 2006;63(8):392-5. 